\title{
La comunidad natural y moral del matrimonio en el derecho natural fichteano de 1796/1797
}

\section{The Natural and Moral Community of Marriage in the Fichtean Natural Right of 1796/1797}

\author{
Héctor Óscar ARRESE IGOR \\ Universidad Pedagógica de Buenos Aires \\ harreseigor@gmail.com
}

Recibido: $16 / 09 / 2012$

Aceptado: 20/02/2014

\section{Resumen}

En este trabajo intento establecer el rol que juega la familia, entendida como una comunidad moral basada en el desarrollo de la virtud y la ternura, en la teoría fichteana del Estado, concebido como una comunidad de ciudadanos egoístas. En primer lugar, reconstruyo la teoría del derecho y el Estado, de la concentración de los tres poderes en el soberano y la teoría de la desobediencia civil, así como sus problemas y contradicciones. Luego analizo la argumentación fichteana para diseñar la figura del matrimonio como una comunidad natural y moral de personas virtuosas. Finalmente, intento mostrar que la familia tiene la función de compensar las deficiencias que el supuesto del egoísmo universal acarrea para la teoría fichteana del Estado.

Palabras clave: derecho, familia, Fichte, matrimonio.

\begin{abstract}
In this paper I try to establish the role which the family, understood as a moral community based on the development of the virtue and the tenderness, plays in the Fichtean theory of the State, conceived as a community of selfish citizens. In the first place, I reconstruct the theory of the right and the State, and the concentration of the three powers in the sovereign, the theory of the civil disobedience, as well as its problems and contradictions. Then I analyze the Fichtean argumentation to design the figure of the marriage as a community of virtuous persons. Finally, I try to show that the family has the function of compensate the deficiencies that the supposition of the universal egoism carries to the Fichtean theory of the State.

Keywords: right, family, Fichte, marriage.
\end{abstract}


Johann G. Fichte desarrolló un proyecto filosófico original para su tiempo, que coincidió con su llegada a la cátedra de Jena en 1794, inaugurada por la publicación del Fundamento de toda la doctrina de la ciencia, donde puso las bases de su idealismo de inspiración kantiana, consistente en una teoría de la subjetividad entendida como autoconciencia y autodeterminación. Dentro de este primer período de su pensamiento, Fichte intentó radicalizar su planteamiento inicial en los cursos que dio entre 1796 y 1799, publicados póstumamente en forma de apuntes de alumnos como Wissenschaftslehre nova methodo.

El proyecto de la Wissenschaftslehre nova methodo va mas allá de la primera propuesta de 1794, en la medida en que Fichte ya no parte de los hechos de la conciencia, para remontarse desde allí al yo trascendental, sino que parte de la autoconciencia práctica del yo, que actúa sobre el mundo. El Fundamento del derecho natural, publicado entre 1796 y 1797, parte justamente de la necesidad de establecer las condiciones de esta autoconciencia práctica y, por lo tanto debe ser entendido en el contexto de estos nuevos desarrollos teóricos de Fichte. En el Fundamento la condición primera de la autoconciencia es el reconocimiento del otro, que viene mediado a su vez por el principio del derecho y permite al yo tomar consciencia de su capacidad de autodeterminación.

Luego de su traslado a Berlín, Fichte continuó revisando su teoría política, tarea que se cristalizó básicamente en los Discursos a la nación alemana (1808), Sistema de la teoría del derecho (1812) y Teoría del Estado (1813). A lo largo del período de Berlín el pensamiento de Fichte fue sufriendo cambios importantes, sobre todo porque dejó de centrarse en el problema de las condiciones de la autoconciencia del yo para buscar la expresión de lo Absoluto en la comunidad político-legal.

En especial la figura de la comunidad familiar fue pasando de ser una reserva moral en un Estado de egoístas racionales, como en el derecho natural de Jena, a constituir un reducto donde se cultiva el interés privado frente a la necesidad de desarrollar el compromiso por el bien común, como ocurre de hecho en los escritos del período de Berlín.

En este trabajo me centraré en los problemas del derecho de familia del Fundamento del derecho natural de 1796/1797, del cual constituye el "Primer Apéndice", mientras que el "Segundo Apéndice" consiste en el derecho cosmopolita. La teoría fichteana del derecho natural está pensada para una comunidad de ciudadanos egoístas, porque no debe recurrir a compromisos morales normativos. Sin embargo, este diseño institucional fundado en el autointerés de los individuos integra a la comunidad moral de la familia, fundada en los lazos de la virtud y la ternura. En este trabajo intentaré determinar el rol que juega la comunidad de la familia en el derecho político fichteano. 


\section{Un Estado para ciudadanos egoístas}

Fichte efectúa una deducción original del derecho, postulándolo como condición de la autoconsciencia. Es decir que el yo no puede ser consciente de sí mismo si no es reconocido por otro yo como un ser racional y libre ${ }^{1}$. Esto es posible a su vez por medio de la acción en la que el otro exhorta al yo a ponerse en marcha para actuar libremente $^{2}$. Pero el yo puede sentirse reconocido sólo si el otro le deja abierta una esfera de acciones libres y no lo coacciona para que actúe de tal o cual manera ${ }^{3}$. En caso contrario, lo estaría tratando como un objeto y no como un sujeto.

\footnotetext{
${ }^{1}$ J. G. Fichte, Grundlage des Naturrechts nach Prinzipien der Wissenschaftslehre, 1796. Citado según la edición de R. Lauth, H. Jacob, Johann Gottlieb Fichte: Gesamtausgabe der Bayerischen Akademie der Wissenschaften, Stuttgart-Bad Cannstatt 1962 ss., con indicación del volumen y de la paginación.; aquí: I, 3, p. 343.

${ }^{2}$ J. G. Fichte, op. cit., I, 3, p. 342. Acerca de este concepto en la Wissenschaftslehre nova Methodo, I Radrizzani, Vers la fondation de l'intersubjectivité chez. Fichte. Des Principes a la Nova Methodo, París, Libraire Philosophique J. Vrin, 1993, pp. 162-164; K. Crone, Fichtes Theorie konkreter Subjektivität. Untersuchungen zur "Wissenschaftslehre nova methodo", Göttingen, Vandenhoeck \& Ruprecht, 2005, pp. 129; sobre su relación con la teoría de Hebert George Mead, J. Stolzenberg, "Fichtes Begriff des praktischen Selbstbewusstseins", en W. Hogrebe, (ed.), Fichtes Wissenschaftslehre. 1794. Philosophische Resonanzen, Frankfurt am Main, Suhrkamp, 1995, pp. 71-95, aquí: pp. 81-82; puede verse una comparación con el caso de los niños lobo de Midnapore, en K. Taver, "Interpersonalität. Von den Wolfskindern zu den Thatsachen des Bewusstseins von 1810/11“, Fichte-Studien, Band 32, 2009, pp. 203-211, aquí pp. 203-204.

${ }^{3}$ J. G. Fichte, op. cit., I, 3, pp. 343-344. E. Cattin, "L'enseignement de l'idéalisme“, en: Kairos, $\mathrm{N}^{\circ}$ 17, 2001, pp. 11-29, aquí p. 18; F. Fischbach, Fichte et Hegel. La reconnaissance, Paris, Presses Universitaires de France, 1999, p. 55; C. Cesa, "Zur Interpretation von Fichtes Theorie der Intersubjektivität“", en M. Kahlo, E. Wolff, R. Zaczyk, (eds.), Fichtes Lehre vom Rechtsverhältnis. Die Deduktion der $\$$ 1-4 der Grundlage des Naturrechts und ihre Stellung in der Rechtsphilosophie, Vittorio Klostermann, Frankfurt am Main, 1992, pp. 53-69, aquí p. 60; E. Düsing, "Sittliche Aufforderung. Fichtes Theorie der Interpersonalität in der WL nova methodo und in der Bestimmung des Menschen “, en A. Mues, (ed.), Transzendentalphilosophie als System, Hamburg, Meiner Verlag, 1989, pp. 174-197, aquí p. 180; Ch. Binkelmann, Theorie der praktischen Freiheit. FichteHegel, Berlín-New York, Walter de Gruyter, 2007, pp. 115-116, Th. Sören Hoffmann, "Staat der Freiheit- freier Staat", Internationales Jahrbuch des deutschen Idealismus, 2, 2004, pp. 221-246, aquí p. 229; respecto del carácter sincrónico y diacrónico de la exhortación, ver F. Oncina Coves, "Das Tempo in Fichtes Jenaer Rechtsphilosophie: der Zeitrhytmus des Rechtsgesetzes", en: FichteStudien, Band 16, 1999, pp. 213-235, aquí p. 223; respecto del trasfondo ilustrado de esta idea, ver G. Zöller, "Kant, Fichte und die Aufklärung", en C. De Pascale, E. Fuchs, M. Ivaldo, G. Zöller, (eds.), Fichte und die Aufklärung, Hildesheim-Zürich-New York, Georg Olms Verlag, 2004, pp. 3552, aquí p. 49. Siep muestra que la renuncia a la coacción del otro no puede ser el único criterio de racionalidad. Más bien se trata de la autolimitación del sujeto en orden a la exhortación del otro y a la producción de un conocimiento en él. Si no, la renuncia a la coacción podría ser producto de una astucia del más débil o del desinterés por la libertad del otro, etc. (L. Siep, Praktische Philosophie im Deutschen Idealismus, Frankfurt am Main, Suhrkamp, 1992, pp. 48-49).
} 
Esto implica que cada uno de los miembros de la relación de reconocimiento debe respetar lo que Fichte denomina como el "principio del derecho" (Rechtssatz), que consiste en que cada uno delimite su esfera de acciones libres en función de dejar disponible otra esfera igual para cada uno de los demás ${ }^{4}$. La razón de la necesidad de introducir al Estado como garante de esta relación de reconocimiento reside en la estructura misma de este vínculo intersubjetivo.

Si el yo puede constituirse como tal en la medida en que es exhortado por el otro a la acción, y esto a su vez es posible sólo si el otro no coacciona al yo para que responda de tal o cual manera, entonces el yo queda libre de actuar como le parezca. Esto implica que el otro no puede estar seguro de si el yo a su vez lo reconocerá o no como un ser libre, es decir de si le dejará también abierta una esfera de acciones libres o no. Cualquiera de las dos respuestas es posible.

Pero la relación de reconocimiento debe ser recíproca, porque el otro también necesita del reconocimiento del yo, dado que también es un ser racional ${ }^{5}$. Por lo tanto, el carácter necesariamente libre y abierto de la relación de reconocimiento constitutiva de la autoconsciencia crea una situación de incertidumbre entre los sujetos. Esto genera inseguridad respecto de la garantía de sus derechos, para lo cual deben delegar su poder en un tercero, esto es en el Estado ${ }^{6}$.

Pero esta fundamentación del Estado afecta a su diseño institucional mismo, porque no puede ser pensado como un Estado para ciudadanos virtuosos, en razón de la incertidumbre misma que le dio origen. Por lo tanto, el diseño debe presuponer el peor de los escenarios posibles, es decir el de un Estado compuesto sólo por ciudadanos egoístas ${ }^{7}$. Esto implica a su vez que el poder debe estar concentrado en manos del gobernante, quien ejerce las funciones ejecutiva, legislativa y judicial ${ }^{8}$. El pueblo no podría participar en la legislación y en la vida política en general, porque su egoísmo pondría en peligro la integridad misma del Estado.

El pueblo delega entonces su poder en el gobernante, para que proteja la libertad y la propiedad de todos, pero necesita reservarse alguna instancia de control del gobierno, dado que también el gobernante puede llegar a actuar por puro egoísmo. Este organismo es el eforato, quien debe monitorear el accionar gubernamental y denunciar públicamente al gobernante, en el caso de que atente contra la constitución, es decir contra el mandato que ha recibido del pueblo.

${ }^{4}$ J. G. Fichte, op. cit., I, 3, p. 358; F. Oncina Coves, op. cit., p. 215.

${ }^{5}$ J. G. Fichte, op. cit., I, 3, p. 344.

${ }^{6}$ J. G. Fichte, op. cit., I, 4, pp. 437-438.

${ }^{7}$ Con palabras de Fichte: "ámate a ti mismo por sobre todas las cosas, y a tus conciudadanos en vista de ti mismo" ("liebe dich selbst über alles, und deine Mitbürger um dein selbst willen"); J. G. Fichte, op. cit., I, 4, p. 69.

${ }^{8}$ J. G. Fichte, op. cit., I, 3, pp. 440-441. 
En este caso, el eforato tiene la obligación de denunciar públicamente lo ilícito y convocar a una asamblea popular, donde el pueblo decidirá destituir o no al gobierno9. Pero puede ocurrir que los éforos mismos sean corruptos, dado que la teoría de Fichte prevé la posibilidad de que todos los ciudadanos sean egoístas, sin excepción. En principio no pareciera haber lugar en la teoría para esta eventualidad, porque todo el poder está en manos del gobierno y los únicos que pueden ponerlo en cuestión son los éforos. Es decir que, si ambos se corrompieran, entonces los ciudadanos quedarían inermes ante una situación tal.

Fichte intenta resolver este problema postulando la posibilidad de que surjan éforos naturales entre los ciudadanos, es decir sujetos que se rebelen contra el estado de cosas e intenten tomar el lugar del eforato y llamar a la rebelión contra el gobierno. Se trataría de una rebelión legítima, porque el poder pertenece originariamente al pueblo, quien meramente lo ha delegado en el gobernante ${ }^{10}$. Pero estos ciudadanos en realidad serían meros delincuentes, de acuerdo con la ley, y no recibirían protección ni apoyo de ninguna institución pública.

Ahora bien, si los ciudadanos son egoístas racionales, entonces apoyarían esta rebelión y se sumarían a ella, como la única manera de proteger su propiedad y su libertad. Si no apoyaran a los éforos naturales, esto sería una clara señal de que el pueblo está tan corrompido como sus gobernantes y éforos. Los éforos naturales se convertirían entonces en verdaderos mártires del derecho, y no tendría sentido siquiera plantearse la construcción de un Estado justo ${ }^{11}$.

Por lo tanto, la teoría de Fichte, al presuponer el egoísmo racional de todos los ciudadanos, queda encerrada en una contradicción, dado que el sistema no garantiza la protección de los derechos de la ciudadanía, en el caso de que el eforato sea corrupto. Pero este diseño del Estado contempla en su interior la existencia de una comunidad moral, que es la familia, donde los esposos se apoyan mutuamente en el desarrollo de la virtud y promueven el progreso de sus hijos en la moralidad. Veamos qué relaciones se dan entre la comunidad jurídica del Estado y la comunidad moral de la familia.

\section{La paradoja de la familia: un enclave de moralidad en medio de un Estado de ciudadanos egoístas}

La familia es una comunidad que, si bien existe dentro del Estado, no es una comunidad legal, porque no es una comunidad constituida por leyes consensuadas entre

\footnotetext{
${ }^{9}$ J. G. Fichte, op. cit., I, 3, p. 450.

${ }^{10}$ J. G. Fichte, op. cit., I, 3, pp. 456-457.

${ }^{11} \mathrm{~J}$. G. Fichte, op. cit., I, 3, p. 458.
} 
sus miembros para regular su coexistencia ${ }^{12}$. Más bien es una comunidad natural, porque está fundada en las condiciones biológicas de sus miembros, y moral en razón de que presupone determinadas virtudes en los cónyuges ${ }^{13}$. Por lo tanto, el derecho de familia no puede establecer los derechos y obligaciones legales que les competen a cada miembro de la familia, si antes no se tiene una idea clara acerca de la constitución de la comunidad matrimonial ${ }^{14}$.

El punto de partida de la deducción del matrimonio consiste en la constatación de que la naturaleza ha puesto en el hombre el impulso sexual, cuya satisfacción permite la reproducción y la conservación de la especie humana ${ }^{15}$. Fichte distingue entre diferentes impulsos naturales, en especial entre aquellos de los cuales los seres humanos no son conscientes (como el hambre o la sed) y aquellos de los que sí lo son. El ser humano puede elegir entre modos alternativos de satisfacer los impulsos naturales de los que es consciente, entre ellos su impulso sexual. El paso de la necesidad a la libertad, de la naturaleza a la moralidad, se da en aquellos impulsos naturales de los que somos conscientes. La posibilidad de elección en la respuesta al impulso sexual del ser humano es el presupuesto que permite a Fichte entender a las relaciones sexuales como relaciones morales ${ }^{16}$. El impulso sexual cumple con la finalidad de la naturaleza aún si es satisfecho sin que intervenga la consciencia reflexiva del ser hu-

${ }^{12}$ J. G. Fichte, op. cit., I, 4, p. 95. Al sostener que el matrimonio no se determina por la ley jurídica, sino por la legislación basada en la naturaleza y la moral, Fichte se opone al positivismo jurídico de la Ilustración (J. Cruz, Cruz, "Amor esclavo. El destino de la mujer en Fichte", Thémata. Revista de Filosofía, $\mathrm{N}^{\circ}$ 9, 1992, pp. 97-112, aquí p. 97). También Fichte se opone a la tesis del positivismo romántico de que el matrimonio es una institución convencional inventada arbitrariamente en un momento determinado de la historia ( J. Cruz Cruz, Fichte. La subjetividad como manifestación del absoluto, Navarra, Ediciones de la Universidad de Navarra- EUNSA, 2003, p. 220).

${ }^{13}$ En este sentido, Honneth argumenta que la familia moderna se ha institucionalizado como una esfera privada separada de la sociedad gracias a tres cambios normativos estructurales: el anclaje de la relación de pareja en una relación de amor, la prohibición del trabajo infantil y la regulación legal del modo en que se comparte la riqueza al interior de la familia (A. Honneth, , "Between Justice and Affection. The family as a field of moral disputes"; en B. Rössler, (ed.), Privacies: Philosophical Evaluations, Stanford, Stanford University Press, 2004, pp. 142-162, aquí p. 145).

${ }^{14}$ En la Rechtslehre el tema del derecho familiar es meramente mencionado y en ningún momento tratado, ya que, a diferencia de la Grundlage des Naturrechts (donde la perspectiva dominante es la del individuo y el derecho familiar regula las relaciones intersubjetivas), se toma distancia de la perspectiva anterior basada en el individuo (C. Senigaglia, "Die Bestimmung des Bürgers beim späten Fichte", Fichte-Studien, Band 24, 2003, pp. 113-126, aquí p. 115).

${ }^{15}$ J. G. Fichte, op. cit., I, 4, p. 96.

${ }^{16}$ B. Frischmann, "Fichte's Theory of Gender Relations in his Foundations of Natural Right"; en T. Rockmore, D. Breazale, (eds.), Right, Bodies and Recognition. New Essays on Fichte's Foundations of Natural Right, Hampshire-Burlington, Ashgate Publishing Company, 2006, pp. 152165 , aquí p. 154. 
mano, esto es, sin que éste tenga una intención clara de colaborar con el plan de la naturaleza. Ahora bien, este impulso puede ser integrado en la vida ética de los hombres, gracias a la mediación del matrimonio. Como veremos más adelante, el matrimonio es la única instancia en la cual los ciudadanos pueden desarrollar las virtudes que corresponden a su sexo.

Pero la reproducción del género humano es posible porque la fuerza procreadora de la naturaleza se dividió en dos mitades complementarias. Se trata de los sexos masculino y femenino, que pueden constituir un ser capaz de autorreproducirse, cuando se reunifican en el acto procreativo. Ahora bien, la naturaleza ha determinado a los sexos, de acuerdo con su función en el acto de la procreación, de modo tal que al sexo masculino le corresponda un rol activo y al femenino uno pasivo. Esto es, el sexo masculino es el principio motor de la procreación, mientras que al sexo femenino le compete la función pasiva de acoger al embrión y alimentarlo hasta que esté en condiciones de nacer ${ }^{17}$. En última instancia, la argumentación descansa sobre supuestos propios de la mentalidad de la época, que Fichte trata como dados de modo natural.

A partir de este análisis de las funciones de los sexos, Fichte concluye que las características del sexo masculino son compatibles con la racionalidad propia del ser humano, mientras que en el caso del sexo femenino esta afirmación podría ser problemática. La razón consiste en una actividad absolutamente espontánea, lo que no entra en contradicción con la satisfacción del impulso sexual masculino, que cumple una función activa. Por el contrario, la satisfacción del impulso sexual femenino consiste en una forma de receptividad y no ya de actividad, lo que entra claramente en conflicto con la índole de la razón. Este conflicto atenta contra el supuesto del que parte Fichte, según el cual la naturaleza tiene una estructura teleológica y, por lo tanto, todo ser vivo debe cumplir con aquella función que le es propia, a fin de promover los fines de aquella totalidad orgánica mayor a la que pertenece.

Si la mujer busca directamente la satisfacción de su impulso sexual, dado que en ello tiene un papel pasivo, iría en contra de su ser racional. Y si por el contrario optara por ser meramente racional y decidiera no satisfacer su impulso sexual, en ese caso la razón se pondría en contradicción con la naturaleza, porque implicaría que la

\footnotetext{
${ }^{17}$ J. G. Fichte, op. cit., I, 4, p. 97 . Fichte presupone la metáfora patriarcal de que la mujer pone sólo el receptáculo (el útero), mientras que el hombre es el único que tiene el papel activo y determinante en la concepción. A este registro pertenece también la metáfora tradicional de la semilla (hombre) y el campo (mujer) (D. Archard,"Familiy Law (First Annex)"; en J. Ch. Merle, (ed.), Johann Gottlieb Fichte. Grundlage des Naturrechts, Klassiker Auslegen, Band 24, Berlín, Akademie Verlag, 2001, pp. 187-196, aquí p. 190). En realidad esta concepción se remonta a Aristóteles, quien sostenía que el esperma es el principio activo de la generación, porque transmite el movimiento y la forma substancial al feto en gestación (Aristóteles, Generación de los Animales 729 b $13-14,736$ b 35).
} 
naturaleza aceptaría y no aceptaría una disposición determinada en un ser vivo particular ${ }^{18}$. Esta enunciación no sólo sería contradictoria en sí misma, sino que también atentaría gravemente contra el supuesto teleológico de Fichte.

Fichte intenta evitar esta contradicción, afirmando que la mujer no debe satisfacer su impulso sexual en tanto que tal, sino más bien bajo la forma de un impulso a una actividad que pertenezca únicamente al género femenino. La mujer no puede satisfacer de modo consciente su impulso sexual y, dado que este impulso puede existir sólo en la medida en que se aparece a la consciencia, la mujer deberá satisfacer intencionalmente otro impulso, esto es el del varón. Al satisfacer el impulso sexual del varón, la mujer no se convierte en un mero medio para su disfrute, porque en última instancia está satisfaciendo el propio impulso sexual, pero de un modo velado ${ }^{19}$. El carácter voluntario de la decisión de satisfacer el impulso del varón es la clave de la estrategia fichteana para intentar salvar la dignidad de la mujer como persona, es decir su carácter de fin en sí mismo, aunque no lo logra porque la mujer retiene un carácter instrumental ${ }^{20}$.

El impulso sexual femenino en su forma natural debe tomar entonces la apariencia de otro impulso, que consiste en entregarse a otro para satisfacer sus necesidades ${ }^{21}$. Se trata del impulso del amor, que juega un rol central a la hora de cuidar de las necesidades de los hijos, bajo la forma de la compasión. Fichte considera que el amor no debe ser considerado como un mero impulso sexual, porque en realidad es el lugar en el que se unen la naturaleza y la razón práctica ${ }^{22}$. Esto implica que, gracias al amor de la mujer, el impulso sexual adquiere una forma moral.

El amor es la más excelente de todas las disposiciones naturales del ser humano, porque responde a la ley moral, que nos exige olvidarnos de nuestro egoísmo y tomar en cuenta a los demás ${ }^{23}$. Se trata de un impulso que sólo puede darse originariamente en la mujer, por las razones biológicas que se han señalado. El amor es la primera forma de autorrestricción de la libertad bruta, y da forma a la primera comunidad,

\footnotetext{
${ }^{18}$ J. G. Fichte, op. cit., I, 4, pp. 97-98.

${ }^{19}$ J. G. Fichte, op. cit., I, 4, p. 100.

${ }^{20}$ B. Frischmann, op. cit., p. 156. Es decir que el amor de la mujer es en realidad una "pasividad activa" (J. Cruz, Cruz, Amor esclavo. El destino de la mujer en Fichte, cit., p. 100). La satisfacción del impulso del amor no consiste en la satisfacción sensible de la mujer, sino la del hombre, por lo tanto la satisfacción femenina es meramente psíquica (J. Cruz Cruz, Fichte. La subjetividad como manifestación del absoluto, cit., p. 229 y 1992, p. 102).

${ }^{21}$ D. Archard, op. cit., p. 188.

${ }^{22}$ No queda claro en qué sentido las mujeres son capaces de actuar de modo racional, dado que Fichte les niega la posibilidad que sí tienen los varones, de actuar con decisión y de acuerdo con conceptos. En las mujeres, por el contrario, la naturaleza "decide" por ellas (B. Frischmann, op. cit., pp. 155-156).

${ }^{23}$ J. G. Fichte, op. cit., I, 4, 100.
} 
que es la familia. El hombre se constituye como ser racional sólo en comunidad, por eso el amor es el origen y la primera forma que toma la razón en el ser humano. Pero la mujer es quien tiene naturalmente la capacidad del amor y quien la origina en los demás (marido, hijos, etc.), por lo cual ella es el origen de la comunidad humana ${ }^{24}$. El varón no tiene el impulso del amor en su naturaleza, sino que lo desarrolla de modo derivado o comunicado por la mujer ${ }^{25}$.

La mujer sólo necesita entonces amar y ser amada por el varón ${ }^{26}$. El amor tiene la forma de la libertad y la actividad, que son compatibles con el carácter activo de la racionalidad que caracteriza al ser humano. Fichte le adjudica a la mujer una naturaleza racional, a diferencia del varón, quien debe adquirir la racionalidad por medio de la educación. H. Schröder considera que esta aparente adulación de la mujer en realidad tiene un carácter represivo, porque la condena al espacio de lo doméstico. La razón de la mujer atiende a lo práctico, al cuidado del otro, a las particularidades de cada situación, etc., lo que la vuelve apta por naturaleza para el cuidado de la casa y la familia. Es decir que la racionalidad de la mujer, si bien es natural, es inferior a la del hombre, que posee una razón especulativa y teorética ${ }^{27}$.

Fichte sostiene que el hombre puede cortejar a la mujer e insinuarse porque su impulso sexual puede manifestarse sin que él sienta vergüenza al respecto. Por el contrario, la mujer debe ser cortejada, porque su pudor natural le impide tomar la iniciativa. Pero el argumento de Fichte fundamenta la validez de iure de la norma de que es el varón quien debe cortejar a la mujer a partir de un hecho, que es el com-

\footnotetext{
${ }^{24}$ D. Morrison, "Women, Family and State in Fichte's Philosophy of Freedom”, en D. Breazale, T. Rockmore, (eds.), New Perspectives on Fichte, New Jersey, Humanities Press International, 1996, pp. 179-191, aquí pp. 185-186.

${ }^{25}$ En sus cartas a Johanna Rahn Fichte enfatizaba siempre la diferencia de géneros entre ambos, es decir su pasión por lograr objetivos en el mundo exterior a la pareja y la superioridad moral de Johanna. La capacidad superior de su esposa para el intercambio emocional provenía según Fichte de su pertenencia al ámbito de lo privado, del intercambio de pensamientos y sentimientos entre las almas (K. Kenkel, "The Personal and the Philosophical in Fichte's Theory of Sexual Difference“, en W. D. Wilson, R. C. Holub, (eds.), Impure Reason. Dialectic of Enlightenment in Germany, Detroit, Wayne University Press, 1993, pp. 278-297, aquí pp. 284-285).

${ }^{26}$ Johanna Rahn también representaba con sus acciones el ideal fichteano de feminidad, dado que mostraba su amor a Fichte a partir de su auto-sacrificio heroico y su sufrimiento a fin de satisfacer los deseos de él. Por ejemplo, en un momento Fichte le expresó a Johanna su deseo de comenzar a reunirse a cenar con un grupo en Jena periódicamente luego de su llegada, a lo que ella respondió epistolarmente que si bien la cocina no era precisamente su pasión, cocinaría con gusto para sus amigos sólo por satisfacer sus deseos (K. Kenkel, op. cit., p. 293; I. Kammerlander, Johanna Fichte; ein Frauenschicksal der deutschen Klassiker, Sttutgart, 1969, p. 50.).

${ }^{27}$ H. Schröder, Die Rechtslosigkeit der Frau im Rechtsstaat. Dargestellt am Allgemeinen Preussischen Landrecht, am Bürgerlichen Gesetzbuch und an J. G. Fichtes Grundlage des Naturrechts, Frankfurt-New York, Campus Verlag, 1979, p. 111.
} 
portamiento de los varones y las mujeres en la sociedad de su época. Por lo tanto es un argumento bastante débil, dado que, en el caso de que fácticamente fueran las mujeres quienes comenzaran a cortejar a los varones, entonces todo el edificio argumentativo caería por su base ${ }^{28}$. Ahora bien, la mujer es libre de degradarse y relacionarse con los varones con el único objetivo de satisfacer su impulso sexual ${ }^{29}$. Pero también pueden intentar elevarse sobre el amor y buscar el éxito personal en lo laboral o en lo político. Fichte considera que ambos extremos son dañinos, porque atentan contra la disposición natural de la mujer hacia el amor.

El amor consiste en la entrega completa que hace la mujer de su personalidad al varón ${ }^{30}$. Por otro lado, sólo en la medida en que la mujer ama al varón de esta manera puede recuperar su personalidad y realizar su identidad femenina. El amor contiene a su vez tres exigencias ${ }^{31}$. En primer lugar, es necesario que la mujer considere al varón como el más digno de amor entre los varones, porque en caso contrario estaría admitiendo que ha satisfecho sólo su impulso sexual, porque se ha entregado al primer o al único varón que ha encontrado, al que a su vez ha utilizado y degradado como persona. En segundo lugar, la mujer debe amar al varón como si su sentimiento fuera eterno. La razón de esto es que la mujer entrega su personalidad, la que no puede cambiar a lo largo de su vida, sino que más bien debe ponerse como idéntica para todo futuro. Finalmente, al entregar la mujer su personalidad, en realidad entrega todo lo que tiene de valioso ${ }^{32}$. Si retuviera algún sentimiento o pensamiento para sí, estaría afirmando que esta parte es más valiosa que su personalidad ${ }^{33}$. Por esta razón la mujer, al casarse, abandona todas sus propiedades y derechos y los coloca en manos de su marido.

Al entregarse a sí misma por medio del amor, la mujer se coloca en una situación de dependencia respecto del varón. Por lo tanto, el carácter moral de la mujer

\footnotetext{
${ }^{28}$ J. Cruz Cruz, Fichte. La subjetividad como manifestación del absoluto, cit., p. 227 y 1992, p. 101. ${ }^{29}$ J. G. Fichte, op. cit., I, 4, p. 98.

30 "Mas en tal donación tiene lugar una secreta alquimia ética, por cuya virtud la mujer recupera su personalidad y su dignidad si 'se entrega por el amor que profesa a este único individuo"' (J. Cruz Cruz, Fichte. La subjetividad como manifestación del absoluto, cit., pp. 230 y J. Cruz, Cruz, Amor esclavo. El destino de la mujer en Fichte, cit., p. 103).

${ }^{31}$ Cf. J. G. Fichte, op. cit., I, 4, pp. 101-102.

${ }^{32}$ B. Duden analiza la idea del "sexo bello", que consiste en que la mujer desarrolla las virtudes necesarias para el ámbito doméstico con naturalidad y no a partir de ningún conflicto interno. En este sentido B. Duden menciona un almanaque que recibió la Sra. Nikolai de una amiga en 1785, donde, en vez de que cada día del año vaya acompañado de la mención de un determinado santo, lo está de una de las virtudes que debe desarrollar la mujer, entre las que están el amor, la dulzura, el ánimo, la paciencia, la inteligencia, la satisfacción con su propio estado, etc. (B. Duden, "Das schöne Eigentum“, en Michel, K., M., Wieser, H., (eds.), Kursbuch 47, Berlín, Rotbuch Verlag, 1977, pp. 137-138).

${ }^{33}$ J. G. Fichte, op. cit., I, 4, p. 102.
} 
sólo podrá desarrollarse adecuadamente si el varón se comporta de una determinada manera. El problema radica en que la mujer sólo puede desarrollar el amor si a su vez está en condiciones de considerar a su marido como el varón más digno de amor de entre todos los de su género. Esto implica que el varón debe ser digno de respeto, lo que exige que éste último desarrolle el impulso natural que le corresponde de acuerdo con su sexo. Mientras que la mujer tiene una disposición natural a entregarse al otro, el varón se caracteriza por la magnanimidad, que consiste en la valentía y la fortaleza de carácter, así como en la condescendencia con los que están bajo su señorío ${ }^{34}$.

Las mujeres suelen despreciar a los varones que son cobardes y no tienen capacidad de decisión, pero no porque busquen seguridad y protección en el varón, sino porque necesitan respetarlo y amarlo. Si el varón busca satisfacer las necesidades de su mujer del mejor modo posible, no lo hace por la necesidad de someterse a sus mandatos, sino de ganarse su estima. La mujer, por su parte, también intentará satisfacer los deseos del varón, pero motivada por el amor. De allí surge la ternura conyugal, que es el sostén del matrimonio, de acuerdo con la cual cada cónyuge encuentra su felicidad en procurar el bienestar del otro ${ }^{35}$.

El ser humano puede progresar desde la naturaleza a la virtud sólo por medio de la relación intersubjetiva que es característica del matrimonio ${ }^{36}$. De este modo, cada persona desarrolla las virtudes que corresponden a su sexo según su impulso natural y a su vez desarrolla, en la medida de lo posible, las virtudes que le corresponden al otro sexo, y que aprende del otro en esta relación de troquelamiento mutuo. Por lo tanto, el matrimonio es una unión entre dos personas de sexos diferentes, que constituye para ellos un fin en sí mismo. De este modo, es la única unión que hace posible el desarrollo pleno de la personalidad de los ciudadanos ${ }^{37}$. El matrimonio, concluye Fichte, es el producto de la unión perfecta entre la naturaleza y la razón.

\footnotetext{
${ }^{34}$ J. G. Fichte, op. cit., I, 4, pp. 102-103.

${ }^{35}$ J. G. Fichte, op . cit., I, 4, p. 103. Fichte sostiene que el matrimonio logra la armonía entre el marido y la esposa en la medida en que cada uno trata de satisfacer las necesidades del otro, pero esto es irrealizable en la estructura jerárquica entre los géneros, donde la mujer termina siendo explotadda por el varón (Frischmann, op. cit., pp. 156-157).

${ }^{36} \mathrm{Al}$ respecto, véase las curiosas afirmaciones de D. Morrison: "el Yo no puede ser un ser humano individual, porque primero debe moverse en la dirección de este Yo, que es un ideal regulativo, en el matrimonio, en el cual todos los elementos físicos y racionales de la humanidad están unidos bajo una única voluntad unificadora" (D. Morrison, op. cit., p. 190).

${ }^{37}$ J. G. Fichte, op. cit., I, 4, pp. 104-105. Cuando Fichte retrata al matrimonio como una unión moral perfecta entre los cónyuges, utiliza el lenguaje de la Empfindsamkeit, de la unión íntima de los cónyuges en una comunidad espiritual, fundada en la ternura recíproca y la intimidad (A. J., La Vopa, Fichte. The Self and the Calling of Philosophy, 1762-1799, Cambridge, Cambridge University Press, 2009, p. 363; 159 y 165).
} 
Una vez que Fichte ha fundamentado el matrimonio como una comunidad natural y moral, debe resolver la cuestión del modo en que deberá ser regulada por el Estado, que debe intervenir en todos los ámbitos en que los ciudadanos coexisten, para proteger su libertad y su propiedad. Sin embargo, el tipo de regulación que debe sufrir el matrimonio debe ser diferente de la que corresponde al derecho coactivo ${ }^{38}$. El motivo de ésto radica en que el matrimonio no es una comunidad legal, porque no es producto de un contrato celebrado por los ciudadanos para proteger su libertad de la intromisión de los demás. Por el contrario, es una comunidad prejurídica que se funda en el impulso sexual de los cónyuges.

Como vimos anteriormente, el matrimonio se funda en el amor que la mujer tiene por el varón, que motiva a su vez a este último a desarrollar la magnanimidad y volverse digno de ser amado ${ }^{39}$. Por lo tanto, el matrimonio no puede existir si la mujer no decide unirse al varón libremente, es decir movida por el impulso del amor. A fin de preservar el matrimonio, el Estado debe velar para que los cónyuges no sean coaccionados para contraer las nupcias. Fichte considera que la mujer es quien debe ser protegida respecto de toda coacción, porque ella es quien sostiene el matrimonio con el amor, mientras que el varón lo desarrollará recién luego de haberse casado y de modo derivado de su esposa, tal como se dijo anteriormente.

Si la función primordial del Estado es proteger la libertad de acción de los ciudadanos, entonces el contrato social debe preservar la libertad de contraer matrimonio según los propios impulsos y deseos ${ }^{40}$. Esta cláusula obviamente impide que la mujer sea obligada por el uso de la fuerza física, porque esto debe ser tipificado como un caso de violación. Por otro lado, la coacción moral y mediada que los padres o parientes ejerzan sobre una doncella, para persuadirla de que contraiga matrimonio con alguien en particular, presenta matices más complejos. Se trata de una forma de violencia moral, que consiste en la utilización de la personalidad de la doncella como medio para los fines de sus padres.

En consecuencia, Fichte sostiene que esta forma de violencia moral es un atentado contra el derecho mismo de ciudadanía, y debe ser juzgado como tal por el Estado. La violencia moral es más perjudicial que la mera violencia física, porque ataca al corazón mismo de la persona, para pervertir su carácter moral por medio de la persuasión y el engaño ${ }^{41}$. De este modo, la mujer pierde la capacidad de amar y, con ella,

\footnotetext{
${ }^{38}$ J. G. Fichte, op. cit., I, 4, p. 106.

${ }^{39}$ Fichte termina sus cartas con elusiones elípticas a sus sentimientos, sin expresarle nunca su amor a Johanna Rahn, mientras que ella siempre es muy clara respecto del tipo de sentimiento que tiene por él. De hecho, Fichte hace constante referencia al amor que Johanna Rahn siente por él, lo cual sustituye a la expresión por escrito de su propio amor. Fichte hace referencia a una falencia en él que es compensada por el amor que Johanna siente por él (K. Kenkel, op. cit., p. 285).

${ }^{40}$ J. G. Fichte, op. cit., I, 4, p. 107.

${ }^{41}$ J. G. Fichte, op. cit., I, 4, p. 108.
} 
su femineidad y su dignidad moral. Fichte sostiene que el Estado debe proteger a esta doncella, quitando a sus padres la custodia y haciéndose cargo de su tutela, hasta que se case por libre decisión. Dado que lo más probable es que la doncella esté acostumbrada a obedecer a sus padres y, en consecuencia, no se anime a querellarlos, el Estado está facultado en estos casos para intervenir de oficio ${ }^{42}$.

Dado que el varón sólo podrá desarrollar el impulso del amor una vez que se ha unido a una mujer y, por derivación, del amor que ella le manifestará, el varón también es perjudicado seriamente si la mujer es obligada a casarse con él ${ }^{43}$. Este matrimonio malogrará su carácter moral y esto le impedirá desarrollar la magnanimidad que le corresponde como varón, porque no tendrá necesidad de hacerse digno del amor de su esposa. Y tampoco podrá desarrollar el amor, porque esta disposición tiene un carácter derivado. Por lo tanto, es del interés de ambos cónyuges que el Estado proteja su libertad de unirse en matrimonio ${ }^{44}$.

De este derecho Fichte deriva la siguiente norma: el Estado debe conocer y confirmar los matrimonios que se celebren entre los ciudadanos. La razón de esta exigencia consiste en que la mejor prueba de que la mujer consiente libremente el matrimonio es su participación en el acto jurídico de la boda, regulada convenientemente $^{45}$. Dado que el matrimonio es una comunidad moral, Fichte considera que debe ser celebrado bajo la supervisión de los funcionarios eclesiásticos, a quienes compete la educación moral del pueblo. Ellos son funcionarios del Estado y, por lo tanto, deben controlar que los esposos no sean presionados de ningún modo. La boda entonces no es la consumación del matrimonio, sino un acto previo orientado simplemente a la protección legal de los interesados.

Ahora bien, el acto jurídico de la boda tiene consecuencias decisivas para la reglamentación y la vigencia del derecho de propiedad de los cónyuges. Fichte sostiene que no puede haber conflicto alguno entre los cónyuges por cuestiones de derecho de propiedad, porque el matrimonio consiste en una vinculación íntima del corazón y la voluntad ${ }^{46}$. En el caso en el que surjan este tipo de pleitos, entonces ya

\footnotetext{
${ }^{42}$ J. G. Fichte, op. cit., I, 4, p. 109.

${ }^{43}$ La mujer es quien puede constituir el matrimonio, ya que el varón puede desarrollar su amor sólo con el tiempo luego de casados. Por esta razón, el varón parece más bien un apéndice en este proceso y sólo puede divorciarse con grandes dificultades (D. Morrison, op. cit., p. 184).

${ }^{44}$ J. G. Fichte, op. cit., I, 4, pp. 109-110.

${ }^{45}$ J. G. Fichte, op. cit., I, 4, p. 110.

${ }^{46}$ J. G. Fichte, op . cit., I, 4, p. 113. Fichte encontró en Johanna Rahn un igual ante quien no sentía vergüenza de confesarle sus apuros económicos, quien no lo humillaría por pasar por tales penurias. En una carta que le mandó desde Leipzig a mediados de mayo de 1789, le confiesa que los gastos del viaje lo han dejado sin un centavo, pero que ella es la única persona a la que puede pedirle ayuda económica, sin sentirse humillado a amenazado por ello. La razón de esto es que la relación con Johanna Rahn estaba exenta de los códigos de honor propios de las relaciones entre los
} 
se ha separado el matrimonio y sólo queda el acto jurídico del divorcio. Cuando el Estado reconoce el matrimonio como una comunidad natural y moral, previa a la comunidad jurídica, entonces deja de considerar a la mujer como una persona jurídica y pasa a reconocer solamente al varón como un sujeto tanto de derechos de propiedad como de derechos civiles. La razón de esto es que, con la celebración del acto jurídico de la boda, el Estado ha reconocido que la mujer decide entregar su personalidad al varón por amor y, de este modo, ha puesto en sus manos sus derechos ${ }^{47}$. El sí del varón, por otro lado, significa su aceptación de la entrega incondicional de la mujer y su compromiso de protegerla y defenderla en el ámbito de lo público.

De aquí en adelante, el varón se convierte en el portavoz jurídico de los derechos de la mujer ante el Estado, como una expresión de su impulso natural a la magnanimidad. Por lo tanto, la idea misma de matrimonio implica que los cónyuges comparten su propiedad, su vivienda, la crianza de los hijos, etc., porque tienen una voluntad común. Dado el carácter natural y moral de esta comunidad, el Estado no tiene atribuciones para regular el modo en que los esposos deben relacionarse. Ahora bien, si el Estado considera a los esposos como una persona jurídica única cuyo representante externo es el varón, todos los demás ciudadanos deben también considerarlos y tratarlos de este modo en sus negocios e intercambios. Dicho de otro modo, en aquellos casos en que haya motivo para litigar o hacer un reclamo contra alguno de los esposos, el ciudadano afectado deberá dirigirse siempre y exclusivamente al varón ${ }^{48}$. A su vez, para que los esposos sean reconocidos por los demás como tales, deberán hacer conocer su matrimonio a todos aquellos con quienes tengan contacto directo ${ }^{49}$.

\section{El rol de la comunidad familiar en la teoría fichteana del Estado}

El impulso natural de la mujer a cuidar de su hijo adopta la forma del sentimiento de compasión, que consiste en experimentar la necesidad del otro como si fuera propia $^{50}$. De este modo, la naturaleza impulsa a la madre a encargarse de la conservación biológica de su hijo. Pero el elemento mediador de la consciencia abre un campo de respuestas que la mujer puede dar al sentimiento natural de la compasión, dado que

\footnotetext{
varones, lo cual los incluía en una comunidad moral íntima entre iguales (A. J. La Vopa, op. cit., p. 160; I. Kammerlander, op. cit., p. 26.).

${ }^{47}$ J. G. Fichte, op. cit., I, 4, p. 114. Ver M. Heinz, F. Kuster, “'Vollkommene Vereinigung'. Fichtes Eherecht in der Perspektive feministischer Philosophie", Deutsche Zeitschrift für Philosophie, 5, 1998, pp. 823-839, aquí pp. 835 y 838 .

${ }^{48}$ La mujer tiene un status púbico vicario, ya que aparece sólo a través del rol público de su marido; no puede votar ni ser elegida para cargos públicos (D. Archard, op. cit., p. 192).

${ }^{49}$ J. G. Fichte, op. cit., I, 4, p. 115.

${ }^{50}$ J. G. Fichte, op. cit., I, 4, p. 138.
} 
puede resistirse o incluso actuar en contra de sus exigencias. Pero, si la mujer actúa en armonía con su naturaleza y se deja llevar por sus impulsos, entonces seguramente cuidará de su niño tanto como de sí misma.

Ahora bien, el varón está unido con la mujer por el lazo de la ternura conyugal, gracias al cual hace propios los fines de la madre. Es decir que la compasión de la madre para con su hijo es la causa de que también el padre se preocupe por su conservación $^{51}$. La comunidad natural y moral del matrimonio es la base entonces de la comunidad natural y moral de la familia.

Pero la educación de los hijos consiste en el desarrollo de su libertad, entendida como el conjunto de sus capacidades para alcanzar los fines que a futuro quieran proponerse. Los padres necesariamente reconocerán a su hijo como un ser racional y lo exhortarán a que se comporte como tal, porque todo ser racional tiende a ver a sus iguales como seres racionales. Por otro lado, la libertad del niño será posible sólo en la medida en que esté protegida su conservación, por lo cual los padres deberán prohibir a su hijo realizar todas aquellas acciones que pongan en peligro su existencia física ${ }^{52}$.

A su vez los padres podrán educar a sus hijos sólo si el Estado les garantiza la autoridad exclusiva para ello y no permite que nadie se inmiscuya en su tarea. Ellos son los únicos que pueden juzgar acerca de los mejores medios para educar a su hijo, por lo tanto es un asunto que debe dejarse librado a su consciencia moral y a su libertad individual. Es decir que tampoco el Estado debe intervenir en este proceso e indicar el curso que debe llevar la formación de los niños.

Sin embargo, esto no significa que el Estado no tenga ningún interés en que sus ciudadanos críen y eduquen a sus hijos. Por el contrario, el Estado necesita que exista una cantidad relativamente estable de habitantes, porque el derecho a la existencia se garantiza por el trabajo de los ciudadanos que producen alimentos y manufacturas. El contrato de protección mismo tiene vigencia sólo si hay una cantidad suficiente de ciudadanos que puedan pagar los impuestos necesarios para el sustento del Estado. Pero esta estabilidad de la cantidad de habitantes es posible sólo si nacen y se incorporan nuevos ciudadanos en reemplazo de los que han fallecido.

Por lo tanto, el Estado tiene atribuciones para coaccionar a los ciudadanos para que formen a sus hijos, de modo tal que el día de mañana puedan servir a los diferentes fines que el Estado exija que ellos promuevan ${ }^{53}$. Por esta razón el Estado no puede permitir que el niño sea tratado por los padres como si fuera de su propiedad (por ejemplo, vendiéndolo como esclavo u obligándolo a trabajar) ${ }^{54}$.

\footnotetext{
${ }^{51}$ J. G. Fichte, op. cit., I, 4, pp. 139-140.

${ }^{52}$ J. G. Fichte, op. cit., I, 4, pp. 140-141.

${ }^{53}$ J. G. Fichte, op. cit., I, 4, p. 142.

${ }^{54}$ J. G. Fichte, op. cit., I, 4, p. 145.
} 
El contrato social que da origen al Estado, y por medio del cual los ciudadanos se comprometen a promover las condiciones de posibilidad del Estado con todos los medios a su alcance, exige entonces que los ciudadanos procreen y eduquen a sus hijos. Fichte considera que el contrato social tiene como un primer momento el contrato de propiedad, por el que los ciudadanos adquieren el compromiso de respetar la propiedad y la libertad de los demás, es decir que se abstienen de invadir su esfera de acciones posibles ${ }^{55}$.

Pero la mera omisión es una condición negativa, y no permite construir una mediación efectiva que garantice efectivamente el derecho de los ciudadanos. Esto amerita la introducción de un segundo momento, constituido por el contrato de protección, que consiste en la cesión de la fuerza de cada uno para aportar a la formación de una potencia común, que sea más fuerte que la de cada ciudadano tomado en particular ${ }^{56}$. De este modo, esta potencia podrá intervenir en el caso de una lesión de derechos y permitirá la vida pacífica en común.

Pero la potencia suprema de la que hablamos debe formarse con el concurso de las capacidades particulares de cada ciudadano. Por esa razón es indispensable la acción de una institución que procree, cuide y eduque a los ciudadanos que luego aportarán su esfuerzo para el bien común. Ahora bien, el egoísmo de los ciudadanos no puede ser el móvil para una tarea como ésta, que no está orientada directamente a la promoción del autointerés, sino más bien al cuidado por las necesidades del niño.

De este modo, la familia se convierte en la instancia privilegiada para esta tarea, gracias al afecto del amor, que surge de la mujer. Por lo tanto, el altruismo de la mujer es uno de los factores que hace posible la formación y la subsistencia del Estado, pensado para ciudadanos egoístas, junto a otros mecanismos reguladores del Estado fichteano, tales como la satisfacción del derecho a la existencia de todos, el derecho de coacción o el eforato ${ }^{57}$.

Fichte tenía el objetivo primordial de construir una teoría del derecho sin ética, para lo que pensó un Estado fundado en el autointerés de los ciudadanos, guiado por el modelo kantiano del gobierno para un pueblo de demonios. Pero, finalmente, las exigencias de su propia teoría lo llevaron inevitablemente a recurrir al altruísmo de la mujer, como madre y esposa, como uno de los sostenes de este sistema legal. Esta contradicción revela en realidad la inconsistencia de su proyecto inicial de construir una teoría del derecho sin ética.

\footnotetext{
${ }^{55}$ J. G. Fichte, op. cit., I, 4, p. 8.

${ }^{56}$ J. G. Fichte, op. cit., I, 4, pp. 9-10.

${ }^{57}$ Desde un punto de vista crítico feminista, H. Schröder sostiene que la fuerza de trabajo de la mujer pone los fundamentos del mismo sistema que luego seguirá utilizándola como esclava (H. Schröder, op. cit., p. 97).
} 